\title{
Relations between uniform connectedness, luminance, and shape similarity as perceptual organizational cues in infancy
}

\author{
Angela Hayden and Ramesh S. Bhat \\ University of Kentucky, Lexington, Kentucky \\ AND \\ Paul C. Quinn \\ University of Delaware, Newark, Delaware
}

\begin{abstract}
Although several studies have examined infants' sensitivity to perceptual organizational cues, few have examined the functional relations among these cues. We examined how uniform connectedness (UC) functions in relation to shape and luminance similarity. UC has been characterized as the entry-level mechanism of perceptual organization and would therefore be predicted to be more salient than the other two cues. We found that UC was more salient than shape similarity organization was, to the point that 6- to 7-month-old infants failed to even organize on the basis of shape in the presence of UC. Luminance similarity, however, was more salient than UC, even though UC was detected by infants in the presence of luminance cues. We conclude that UC is not necessarily the most salient mechanism of perceptual organization in infancy. Moreover, the luminanceUC-shape salience hierarchy exhibited by 6- to 7-month-olds in the present study is consistent with the order of development of sensitivity to these organizational cues.
\end{abstract}

Perception of the world around us seems effortless and straightforward, but our visual system is not as simple as we may think. It has to organize the display of colors, luminances, connected surfaces, and other properties of light available in the environment into meaningful objects and relations that allow us to function adaptively. Wertheimer (1955) proposed several Gestalt principles of organization (similarity, good continuation, common fate, closure, and proximity) to explain how humans organize their visual world. Subsequent investigations suggested that organization takes place very early in visual processing, most likely preattentively and prior to object recognition (e.g., Duncan, 1984; Duncan \& Humphreys, 1989; Kahneman \& Henik, 1981; Kahneman \& Treisman, 1984; Moore \& Egeth, 1997; Neisser, 1967; Pomerantz, 1981; Treisman, 1986).

This view of perceptual organization became the conventional wisdom as taught in introductory psychology and perception texts, but has been challenged within the last 15 years by Palmer and others. Palmer and Rock (1994) argued that organization based on these principles does not occur as early in vision as was previously assumed. Citing studies that they and others have conducted showing that the perception of depth, illusory figures, and lightness constancy takes place prior to grouping, Palmer and Rock contended that there must be an even more primitive perceptual phenomenon that allows humans to perceive the elements themselves prior to organizing them. They suggested that this phenomenon is uniform connectedness (UC). Palmer and Rock described the concept of UC as follows: "[a] connected region of visual properties - such as luminance or lightness, color, texture, motion, and possibly other properties - strongly tends to be organized as a single perceptual unit" (p. 30). Palmer and Rock argued that grouping cannot take place without uniformly connected elements. In support of Palmer and Rock's theory, Kramer and Watson (1996; Watson \& Kramer, 1999) found that perceptual judgments were more rapid when involving a single $\mathrm{UC}$ region than when involving multiple regions.

Other research indicates that UC may not be as salient as Palmer and colleagues suggested. These studies, in general, have found that the detection of a uniformly connected pattern (one piece of an object literally connected to another) is no more rapid than the detection of disconnected elements that can be organized on the basis of other Gestalt principles of organization. For instance, Han, Humphreys, and Chen (1999) found that UC did not speed the processing of a scene that was already organized on the basis of proximity, but it did speed the processing of organization on the basis of shape. Han et al. concluded that, although UC may be more salient than shape for perceptual organization, it may not be any more salient than proximity (see also Kimchi, 1998, 2000).

R.S.Bhatt, rbhatt@email.uky.edu 
Infant research directly examining sensitivity to UC has been quite limited. Hayden, Bhatt, and Quinn (2006) habituated infants to a stimulus containing uniformly connected figures (the figures resembled solid red barbells), and then tested them with a connected barbell paired with a disconnected barbell (the connecting bars were rotated until the circles that made up the ends of the barbells were no longer connected). Three- to 4-month-old infants looked longer at the disconnected pattern than at the familiar connected bar, a result that was shown in a control condition not to be attributable to an a priori preference, suggesting that sensitivity to UC as an organizing principle is present at this early age.

Most infant research, even the Hayden et al. (2006) study, has focused on the development of the ability to organize on the basis of a specific principle of organization such as good continuation (Quinn \& Bhatt, 2005a), lightness similarity (Quinn, Burke, \& Rush, 1993), and form similarity (Quinn, Bhatt, Brush, Grimes, \& Sharpnack, 2002). However, virtually nothing is known about how these principles of organization function in relation to each other in infancy. It is essential to look at such relationships, because there are many different cues and rules that could be used in organizing the perceptual world, and it is important to know how they function in concert with one another, especially when different cues point to different organizations. Moreover, rapid changes in perception occur during infancy, and, to completely understand the development of perceptual organization, it is critical to explore how these grouping principles function together (or fail to function) in an infant's perceptual field.

Quinn and Bhatt (2006) explored the differential readiness to organize on the basis of luminance versus shape similarity. When habituated to solid horizontal or vertical bars, 6- to 7-month-old infants tested with light and dark squares arranged in alternating columns or rows of luminance preferred squares arranged in a novel orientation over squares arranged in the orientation to which they had been habituated; that is, infants transferred organization on the basis of bar orientation to organization on the basis of the arrangement of light/dark luminance elements. However, infants failed to show a preference in this same procedure when familiarized to bars and tested with columns or rows of shapes (i.e., columns or rows of Xs and Os). In other words, infants were able to generalize from solid horizontal or vertical bars to columns or rows of individual elements on the basis of luminance similarity but not shape similarity. In contrast, 6- to 7-month-olds were able to organize on the basis of both luminance and shape elements if they were exposed to columns/rows of elements during familiarization and tested with bars (Quinn \& Bhatt, 2006; Quinn et al., 2002). Therefore, exposure during habituation was necessary for 6- to 7-month-olds to organize on the basis of shape, but it was not necessary for luminance-based organization. This study thus suggests that luminance similarity may be more readily accessible, and thus more important to infants, than shape similarity is. However, Quinn and Bhatt (2006) did not directly address the issue of differential significance of cues. That is, they did not examine whether for infants, as for adults, there are differences in the salience of cues concurrently present in images. ${ }^{1}$ This is the issue addressed in the present experiments.

Palmer and Rock (1994) suggested that UC determines the basic entry-level units into perception. It would seem appropriate to begin with UC and test its salience in relation to two grouping principles that have already been tested with infants. In the following series of experiments, therefore, we explored the importance of UC when compared with shape and luminance similarity.

\section{EXPERIMENT 1A}

As noted above, Han et al. (1999) found that UC takes some precedence over shape similarity for adults. Thus, in Experiment 1A, we examined whether UC is more salient than shape-based organization in infancy also. Three- to 4-month-olds in Quinn and Bhatt (2005b) utilized shape similarity to organize information when "trained" to ignore the specific shapes and to attend to the overall organization of the image. Such "training" is unnecessary for 6- to 7-month-olds to exhibit sensitivity to shape similarity organization (Quinn et al., 2002). Specifically, Quinn et al. (2002) found that 6- to 7-month-olds, but not 3- to 4-month-olds, were sensitive to shape organization when habituated to rows or columns of Xs and Os and tested with vertical and horizontal bars. In the follow-up study (Quinn \& Bhatt, 2005b), 3- to 4-month-olds were familiarized with trial-to-trial variation in the shapes depicting the row or column organization during familiarization $(\mathrm{X}-\mathrm{O}, \mathrm{H}-\mathrm{I}$, and square-diamond), and now organized by shape similarity. These results demonstrate that shape similarity is more tenuous at 3 to 4 months of age than at 6 to 7 months. However, as described above, even 6- to 7-month-olds have difficulty organizing images on the basis of shape similarity under certain circumstances (i.e., when they have not had an opportunity to experience the individual shapes during familiarization; see Quinn \& Bhatt, 2006). Therefore, the ability to organize on the basis of shape similarity improves as infants develop, but even older infants fail to organize in particular task contexts, suggesting that it is not a completely robust phenomenon, even at 6 to 7 months of age. In contrast, 6- to 7-month-olds had no difficulty organizing on the basis of UC in Hayden et al. (2006). These results suggest that UC may be more salient than shapebased organization at 6 to 7 months of age. We tested this hypothesis in the present experiment.

Infants in this experiment were habituated to a pattern which could be organized on the basis of both UC and shape (see Figure 1). The stimuli contained alternating rows or columns (counterbalanced) of two different shapes (Xs and Os). These shapes were connected by a thick black bar in the same configuration (rows or columns) in which the shapes were organized. In other words, infants were habituated to patterns in which both UC and shape similarity signaled the same organization (rows or columns). Following habituation, infants were tested with a pair of new stimuli: one in which the shape organization was altered, and the other in which the UC was altered (see Figure 1). If one of the perceptual organizational cues 


\title{
$90^{\circ}$ Rotation Condition
}

A

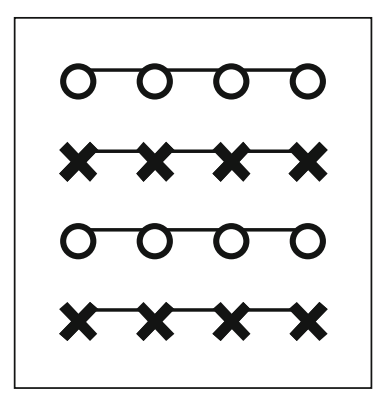

Habituation Stimulus
B

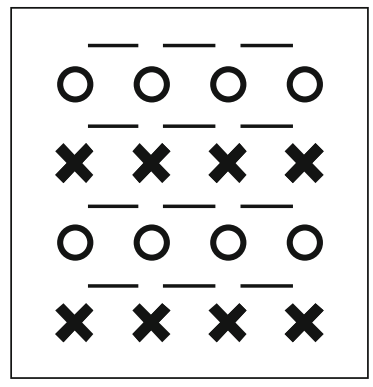

Change in UC
C

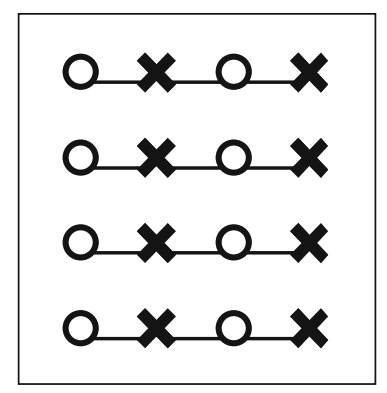

Change in Shape

\section{Random Condition}

B

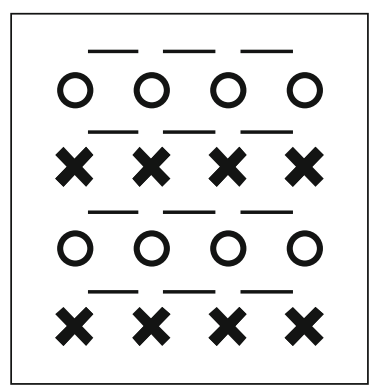

Change in UC
D

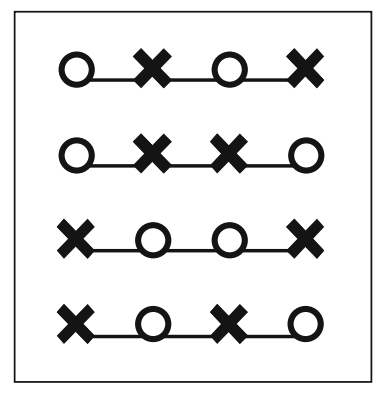

Change in Shape

Habituation Stimulus

\begin{abstract}
Figure 1. Examples of the stimuli used in Experiment 1A. The examples shown here involve habituation stimuli that are organized in rows. Half of the infants were habituated and tested with these stimuli. The other half were habituated and tested with corresponding patterns in which the habituation stimuli were arranged in columns. Infants were habituated with patterns of the kind shown in panel A. These patterns could be organized on the basis of both uniform connectedness (UC) cues and shape similarity cues. They were tested with a pattern in which the $U C$ was altered (panel B) paired with a pattern in which the shape similarity was altered (panel C or D, depending on condition).
\end{abstract}

were more salient to infants than the other, the change induced by the manipulation of this cue should be more novel to infants and they should look longer at this pattern than at the pattern in which the less salient cue was altered. Since shape similarity organization seems to have a protracted developmental timeline compared with UC, we tentatively predicted that $\mathrm{UC}$ would be more salient than shape-based organization in Experiment 1A. Palmer and Rock's (1994) theory on the importance of UC as well as the adult reaction time (RT) study conducted by Han et al. (1999) would predict similar results.

\section{Method}

Participants. Twenty-four 6- to 7-month-olds (13 males, $11 \mathrm{fe-}$ males; mean age $=200.67$ days, $S D=12.29$ ) participated. They were predominantly Caucasians from middle-class families, and were recruited from birth announcements in the local newspaper as well as by word of mouth.

Stimuli. The habituation stimuli used in this experiment were created using two different principles of organization: shape similarity and UC. Shape similarity organization was achieved by combining four rows (or columns, depending on the counterbalanced condition) of alternating Xs and Os (see Figure 1). The basic patterns mirrored the patterns used in the Quinn et al. (2002) study. The shapes in the rows or columns were uniformly connected by 1.78 -mm-thick black lines running in the same direction as the shape organization (i.e., if shape organization were in columns, the UC organization would be, too). The habituation patterns subtended roughly $11.31^{\circ}$ both horizontally and vertically. The midpoint distances between the individual shapes subtended roughly $3.24^{\circ}$ in both the vertical and horizontal directions. Individual Xs and Os subtended roughly $1.65^{\circ}$.

The two test patterns in each condition were created by altering one type of organization (UC or shape) while the other remained intact. A stimulus with a UC change had the thick black bars above (or adjacent to, depending on whether the organization was rows or columns) and disconnected from the shapes (see Figure 1). The bars were moved $7 \mathrm{~mm}$ total and were $5 \mathrm{~mm}$ above or beside the shapes (depending on whether the infant was habituated to a column or row orientation pattern). In total, the bars' displacement subtended $0.89^{\circ}$ in the test stimulus. This change altered the UC organization of the image, because the rows and columns were no longer uniformly connected. The shape organization in this stimulus remained stable. The bars in the shape-altered stimulus were moved a distance equivalent to those in the UC change pattern, but to maintain UC the bars remained connected to the shapes. This was done to ensure that, if infants preferred the UC-altered image to the shape-altered image, 
this preference was not merely due to the movement of the bars but rather to the change in UC; in other words, we equated the amount of displacement in the connecting bars in the shape change and UC change test stimuli.

The change in UC could be thought of as an alteration of one type of organization to another (i.e., from connected to disconnected), or it could be thought of as a change from an organized pattern to an unorganized pattern (i.e., from connectedness to a break in connectedness). In order to equate for these two ways of conceptualizing the change as much as possible, we believed that it was important to test infants with two different versions of altered shape organization. In the $90^{\circ}$ rotation condition, infants were tested with a shape pattern that was rotated $90^{\circ}$, turning columns into rows and vice versa (see Figure 1). This change altered the shape pattern from one organization to another (rows to columns, or vice versa). The UC organization remained the same (rows were still rows, columns were still columns) even though, as mentioned above, the bars connecting the blocks were moved. In the "random condition," infants were tested with an unorganized shape configuration: The Xs and Os were randomly dispersed throughout the image in a manner similar to the stimuli employed in Experiment 2 of the Quinn et al. (2002) study.

Apparatus. The stimuli were displayed on a 20-in. IBM computer monitor. Each infant sat on its mother's lap, approximately $45 \mathrm{~cm}$ in front of the monitor. Black ceiling-to-floor screens ensured that the infants could see the monitor only during the experimental session. A video camera, located on top of the monitor, recorded each infant's fixations.

Procedure. A habituation/novelty preference procedure was used for this study (e.g., Bhatt \& Bertin, 2001). Prior to the beginning of the trial, an attention getter (a rapidly pulsating red-blue-green pattern) was presented in the center of the screen. The experimenter pressed a key to begin the trial when the infant looked toward the attention getter. Following the keypress, two identical habituation patterns were presented, one on each side of the screen. These patterns remained on the screen until the infant looked away for $2 \mathrm{sec}$ or more, or if $60 \mathrm{sec}$ had elapsed with the infant looking at the patterns. These trials continued until the infant's looking time average over three consecutive looking trials had decreased to $50 \%$ of the looking time average on the first three trials (or until a maximum of 20 habituation trials had been completed). Following habituation, infants were tested for their preference between the two different organization changes in two 10 -sec test trials. The initial left-right presentation of the stimulus pairs was counterbalanced across participants. The positions of the test stimuli were switched between test trials as well. As noted above, the type of shape test that infants saw was counter- balanced across participants; half viewed the $90^{\circ}$ rotation stimuli, and the other half viewed the completely scrambled stimuli.

Infant looking times were coded offline by an experimenter unaware of the location of the test patterns. The DVD was reduced to $25 \%$ of regular speed for coding. To examine the reliability of coding, the performance of 10 randomly chosen participants was coded by another naive experimenter. The Pearson correlation between the 2 observers' scores was .98 .

\section{Results and Discussion}

Table 1 displays infant look durations for the habituation trials. A trial (first three, last three) $\times$ condition $\left(90^{\circ}\right.$ rotation, random) ANOVA performed on the look duration data during habituation indicated a main effect of trial $[F(1,22)=70.91, p<.0001]$. Neither the main effect of condition nor the trial $\times$ condition interaction was significant (all $p s>.05$ ). Therefore, infants were habituated prior to the test trials, and there were no differences between the two conditions.

Preference during the test trials was assessed by computing a percentage score that indicated preference for the disconnected pattern. This score was computed by dividing the total looking time to the disconnected pattern by the total looking time to both patterns and multiplying this ratio by 100 . If infants found one type of organizational change to be more salient than another, they should clearly prefer to look more at the pattern that exhibits this change than at the alternate pattern, and should exhibit a preference score significantly different from $50 \%$.

Infants' preference scores are displayed in Table 1. An independent-samples $t$ test revealed no difference between the two shape disruption conditions $\left[90^{\circ}\right.$ rotation and random conditions; $t(22)=-0.76, p>.20$, two-tailed]. Therefore, we combined these conditions for our final analysis. Infants preferred to look at the pattern displaying a change in UC cues significantly more than at a pattern displaying a change in shape cues $[t(23)=2.95, p<.01$, two-tailed].

The results of this experiment suggest that UC is more salient than shape similarity organization, as Palmer and

Table 1

Mean Fixation Times, in Seconds, During the Habituation Trials

in Experiment 1A and Preference Scores, in Percentages, for the

Connectedness Change Pattern During the Test Trials in Experiment $1 \mathrm{~A}$ and Spontaneous Preference Trials in Experiment $1 B$

\begin{tabular}{|c|c|c|c|c|c|c|c|}
\hline & \multicolumn{4}{|c|}{ Habituation Trials } & \multirow{2}{*}{\multicolumn{3}{|c|}{$\begin{array}{c}\text { Test Trials: } \\
\text { Preference for } \\
\text { Connectedness } \\
\text { Change Organization }\end{array}$}} \\
\hline & \multicolumn{2}{|c|}{$\begin{array}{c}\text { First } \\
\text { Three Trials }\end{array}$} & \multicolumn{2}{|c|}{$\begin{array}{c}\text { Last } \\
\text { Three Trials }\end{array}$} & & & \\
\hline & $M$ & $S D$ & $M$ & $S D$ & $M$ & $S D$ & $t$ \\
\hline \multicolumn{8}{|l|}{ Experiment $1 \mathrm{~A}$} \\
\hline $90^{\circ}$ rotation & 24.13 & 12.23 & 9.55 & 5.80 & 56.74 & 11.82 & $1.98^{*}$ \\
\hline Random & 25.64 & 14.07 & 7.51 & 3.77 & 61.46 & 18.02 & $2.20^{* *}$ \\
\hline Combined & 24.88 & 12.92 & 8.53 & 4.90 & 59.10 & 15.10 & $2.95^{* * *}$ \\
\hline \multicolumn{8}{|l|}{ Experiment 1B } \\
\hline $90^{\circ}$ rotation & & & & & 53.70 & 11.30 & 1.13 \\
\hline Random & & & & & 52.38 & 11.62 & 0.71 \\
\hline Combined & & & & & 53.04 & 11.23 & 1.33 \\
\hline
\end{tabular}


Rock's (1994) theory would predict. Infants exhibited a preference for the change in UC organization over the change in shape organization in both the $90^{\circ}$ rotation and random conditions. These data mirror the results of the Han et al. (1999) study, which showed similar outcomes with adults, using RT measures. Thus, in infancy, as in adulthood, UC is more salient than shape similarity as a perceptual organizational cue.

\section{EXPERIMENT 1B}

There is a potential alternative explanation for the results obtained in Experiment 1A: Infants' performance during the test may have been due to a priori preferences between the test patterns instead of resulting from the differential salience of the changes from habituation to test of the UC organization versus shape similarity organization. To examine this issue, in Experiment 1B we tested a new group of infants with the test stimuli used in Experiment $1 \mathrm{~A}$ but without any habituation experience. If these infants exhibited a preference similar to that exhibited by the infants in Experiment 1A, our conclusion in Experiment $1 \mathrm{~A}$ about the relative salience of $\mathrm{UC}$ and shape similarity would be suspect. If, on the other hand, infants failed to exhibit a spontaneous preference between the test stimuli, their performance in Experiment 1A would probably be due to the higher salience of UC over shape similarity cues.

\section{Method \\ Participants. Twenty-four 6- to 7-month-olds (14 males, $10 \mathrm{fe-}$ males; mean age $=199.50$ days, $S D=10.09$ ) participated. \\ Procedure. The procedure was exactly the same as that used in Experiment 1A, except that infants did not experience habituation trials and were directly tested on two 10 -sec test trials.}

\section{Results and Discussion}

Infants' preference scores are displayed in Table 1. A condition $\times$ nature of test (habituation in Experiment 1A, spontaneous preference in Experiment 1B) ANOVA failed to reveal any significant main effects or interactions (all $p$ s > .05). Consequently, we combined the data from the two conditions in our main analyses. Infants failed to exhibit a preference during the spontaneous preference test. Their mean preference score was not significantly different from the chance level of $50 \%[t(23)=1.33$, $p>.20$, two-tailed]. Also, an independent-samples $t$ test revealed that the mean preference score exhibited by the infants in the spontaneous preference condition of Experiment 1B was marginally different from the novelty preference score exhibited by the infants in Experiment $1 \mathrm{~A}$ $[t(46)=1.58, p<.06$, one-tailed]. This marginal difference implies that some caution should be exercised in drawing conclusions. However, the failure of infants to exhibit a greater than chance level of discrimination in Experiment 1B, combined with a statistically significant discrimination in Experiment 1A, suggests that infants' performance in Experiment 1A was not due to a priori preferences between the test stimuli but to the greater sa- lience of changes in UC-based rather than shape-based organization.

\section{EXPERIMENT 2}

The greater salience of UC over the shape similarity organization in Experiment $1 \mathrm{~A}$ leads to the question of whether the 6- to 7-month-old infants found the UC changes more compelling, even though they were sensitive to the shape similarity changes during the test, or whether the shape similarity changes were not even perceived because of the presence of UC cues. As mentioned above, shape similarity organization is perceived without the presence of UC cues (Quinn et al., 2002). However, under some challenging circumstances (Quinn \& Bhatt, 2006), infants of this age have difficulty organizing an image on the basis of shape similarity. To examine whether the presence of UC makes shape cues more challenging for 6- to 7-month-olds to detect, 24 infants were habituated to the same stimuli used in Experiment 1A, then tested with patterns in which only the shape similarity organization was altered.

\section{Method}

Participants. Twenty-four 6- to 7-month-olds (12 males, $12 \mathrm{fe}-$ males; mean age $=202.50$ days, $S D=10.82$ ) participated.

Stimuli. The habituation stimuli in this experiment were identical to the habituation stimuli in Experiment 1A. Infants were tested with the habituation stimulus paired with the shape-organizationaltered stimuli from Experiment 1A (half of the infants saw the $90^{\circ}$ rotation change and half saw the random organization change). In other words, UC remained the same in the test patterns as in the habituation patterns; only shape similarity organization changed (see Figure 2 for examples).

Procedure. The procedure was identical to that of Experiment 1A.

\section{Results and Discussion}

Table 2 displays infant look durations for the habituation trials. A trial $\times$ condition ANOVA was performed on the look duration data during habituation. Results indicated a main effect of trial $[F(1,22)=52.98, p<.0001]$. Neither the main effect of condition nor the trial $\times$ condition interaction was significant (both $p \mathrm{~s}>.05$ ). Therefore, infants were habituated prior to the test trials, and there were no differences in habituation between the two conditions.

Infants' preference scores for the novel shape-organization patterns are displayed in Table 2. An independent-samples $t$ test revealed no difference between the two shape disruption conditions $\left[90^{\circ}\right.$ rotation and random conditions; $t(22)=$ $0.87, p>.20$, two-tailed]. Thus, in the following analysis, both conditions were combined. Infants failed to show a preference for the novel shape-organized pattern over the habituation pattern $\left[t(23)=-0.03, p>.20\right.$, two-tailed]. ${ }^{2}$

Infants in this experiment were unable to detect a change in shape organization in the presence of UC. Research has shown that infants of this age have the ability to detect shape organization in the absence of UC cues, however (Quinn et al., 2002). In fact, the stimuli used in this experiment were identical to those used in the Quinn et al. (2002) study, aside from the thick bars that connected the 


\section{$90^{\circ}$ Rotation Condition}

E

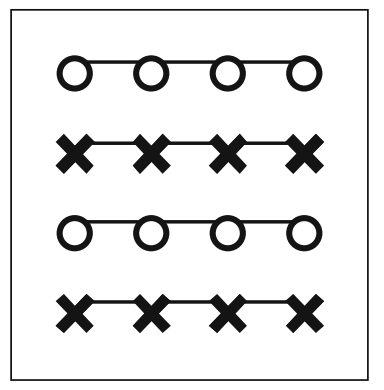

Habituation Stimulus
$\mathbf{E}$

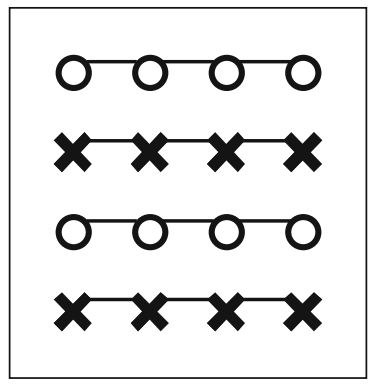

Habituation Stimulus
$\mathbf{F}$

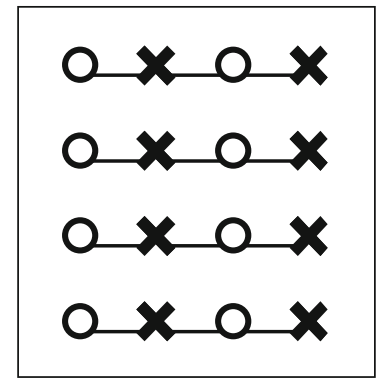

Change in Shape

\section{Random Condition}

$\mathbf{E}$

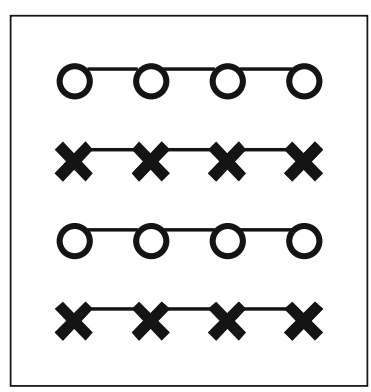

Habituation Stimulus
G

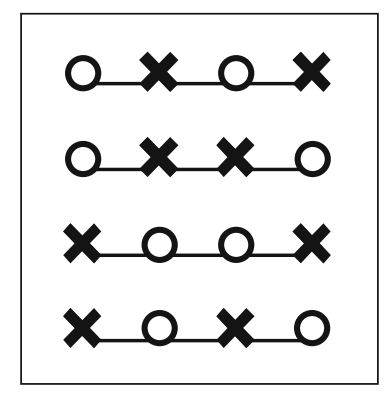

Change in Shape

Figure 2. Examples of stimuli used in Experiment 2. Infants were habituated to patterns of the kind shown in panel $E$ (which can be organized on the basis of both $U C$ and shape similarity cues) and then tested with a pattern of the kind shown in panel $E$ paired with a pattern of the kind shown in panel $F$ or G, depending on condition.

shapes. This comparison suggests that $\mathrm{UC}$ is such a strong organizational cue for 6- and 7-month-olds that the presence of these cues makes detection of shape organization cues difficult. The results of Experiment 2 are also consistent with the findings by Quinn and Bhatt (2006) that shape similarity organization is not robust at 6 to 7 months of age. Organization on the basis of shape can be completely disrupted by a stronger organizational cue. In the next experiment, we explored the relative importance to $\mathrm{UC}$ of a more robust organizational principle.

Table 2

Mean Fixation Times, in Seconds, During the Habituation Trials and Mean Preference Scores, in Percentages, for the Novel Shape Change Pattern in Experiment 2

\begin{tabular}{|c|c|c|c|c|c|c|c|}
\hline & \multicolumn{4}{|c|}{ Habituation Trials } & \multirow{2}{*}{\multicolumn{3}{|c|}{$\begin{array}{c}\text { Test Trials: } \\
\text { Preference for Novel } \\
\text { Shape Organization }\end{array}$}} \\
\hline & \multicolumn{2}{|c|}{$\begin{array}{c}\text { First } \\
\text { Three Trials }\end{array}$} & \multicolumn{2}{|c|}{$\begin{array}{c}\text { Last } \\
\text { Three Trials } \\
\end{array}$} & & & \\
\hline & $M$ & $S D$ & $M$ & $S D$ & $M$ & $S D$ & $t$ \\
\hline $90^{\circ}$ rotation & 22.52 & 13.88 & 7.39 & 3.44 & 52.04 & 14.91 & $0.475^{\circ}$ \\
\hline Random & 22.52 & 14.03 & 8.38 & 6.71 & 47.83 & 7.94 & $-0.948^{\circ}$ \\
\hline Combined & 22.52 & 13.65 & 7.89 & 5.24 & 49.94 & 11.88 & $-0.027^{\circ}$ \\
\hline
\end{tabular}

${ }^{\circ} p>$.20, two-tailed, n.s.

\section{EXPERIMENT 3A}

In Experiments 1A and 2, UC cues dominated shape organization for 6- and 7-month-olds so completely that shape organization was not even detected in the presence of UC cues. For this next experiment, we used luminance similarity, a cue arguably more robust at 6 to 7 months of age, in order to further explore the importance of UC. The ability to organize on the basis of luminance may be present even at birth (Farroni, Valenza, Simion, \& Umiltà, 2000; but see Quinn \& Bhatt, 2006). There is consistent evidence that infants have the ability to group on the basis of luminance by at least 3 to 4 months of age (Quinn \& Bhatt, 2006; Quinn et al., 1993). When familiarized to a display of columns or rows of light and dark colored squares, 3- to 4-month-olds looked longer at a novel display in which the rows or columns had been rotated $90^{\circ}$ than at the familiar display (Quinn et al., 1993). In contrast to shape organization (which, as discussed above, undergoes development between 3 and 6 months of age; see Quinn \& Bhatt, 2005b), luminance organization seems to be fairly well developed by at least 3 months of age, if not earlier. Luminance organization is also more readily available (and 
thus may be more entry level) than shape similarity organization at older ages (Quinn \& Bhatt, 2006). Thus, although UC cues were more salient than shape-based organization was in Experiments 1A and 2, it is not clear whether UC will also be more salient than luminance-based organization - given the robustness of the latter in infancy.

As in Experiment 1A, infants in the present experiment were first habituated to a stimulus that could be organized on the basis of two different organizational cues (UC and luminance similarity). They were then tested with a pair of stimuli, in each of which one organizational cue was altered and the other remained stable. Our expectation was that if one type of organizational change were more salient than another, infants would prefer the pattern with the change in that particular organization over the one containing an organizational change less apparent to them.

\section{Method}

Participants. Twenty-four 6- to 7-month-olds (12 males, 12 females; mean age $=199.33$ days, $S D=11.29$ ) participated.

Stimuli. Familiarization stimuli were similar to those used in Experiment $1 \mathrm{~A}$ (see Figure 3). The patterns were organized into col- umns or rows of filled and unfilled squares uniformly connected by a thick black bar. The habituation patterns subtended roughly $11.68^{\circ}$ both horizontally and vertically. The midpoint distances between the individual squares subtended roughly $3.24^{\circ}$ in both the vertical and horizontal directions. The squares subtended roughly $1.65^{\circ}$.

As in Experiment 1A, the test stimuli were such that, in each pattern, one type of organization was altered and the other type of organization kept intact. The bars were moved above or beside the squares in the novel UC stimulus (dependent on the vertical or horizontal orientation of the habituation stimulus), and the luminance organization was rotated $90^{\circ}$ or randomized in the novel luminance pattern (see Figure 3 for examples).

Procedure. The procedure was the same as that used in Experiment $1 \mathrm{~A}$.

\section{Results and Discussion}

Table 3 displays infant look durations for the habituation trials. A trial $\times$ condition ANOVA indicated a main effect of trial $[F(1,22)=33.83, p<.0001]$. Neither the main effect of condition nor the trial $\times$ condition interaction was significant (both $p \mathrm{~s}>.05$ ). Therefore, infants were habituated prior to the test trials, and there were no differences in habituation between the two conditions.

\section{$90^{\circ}$ Rotation Condition}

H

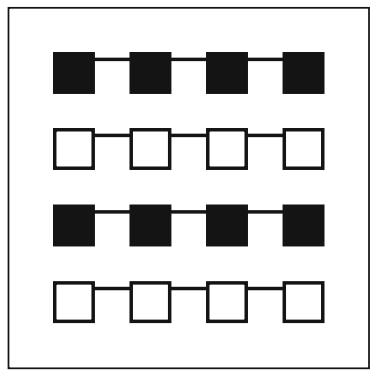

Habituation Stimulus

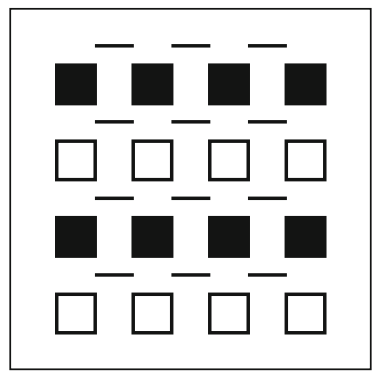

Change in UC
$J$

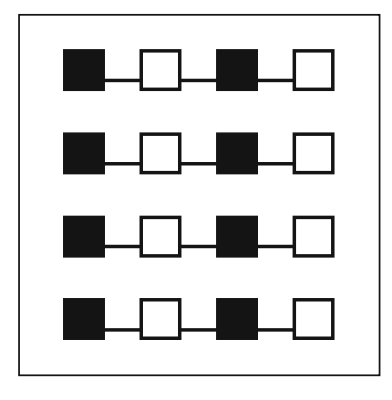

Change in Luminance

\section{Random Condition}

H

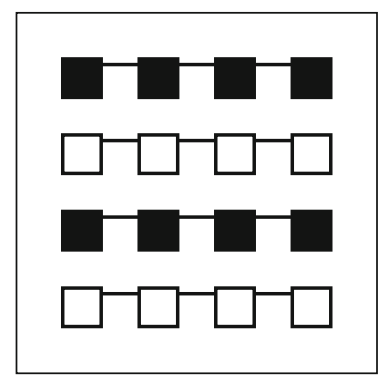

Habituation Stimulus
I

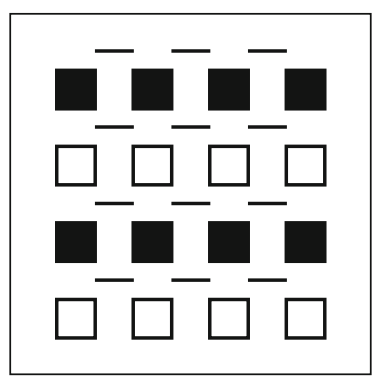

Change in UC
$\mathbf{K}$

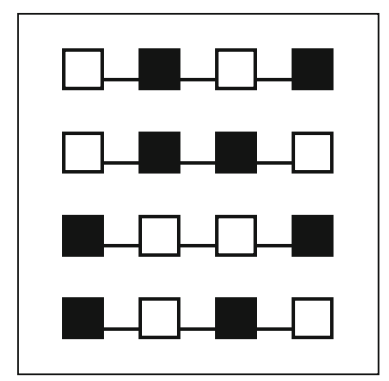

Change in Luminance

Figure 3. Examples of the stimuli used in Experiment 3A. The examples shown here involve habituation stimuli that are organized in rows. Half of the infants were trained and tested with these stimuli. The other half were trained and tested with corresponding patterns in which the habituation stimuli were arranged in columns. In Experiment $3 \mathrm{~A}$, infants were habituated with a pattern (of the kind shown in panel $\mathbf{H}$ ) that was composed of both $U C$ and luminance similarity cues. They were tested with a pattern in which the UC was altered (panel I) paired with a pattern in which the luminance similarity was altered (panel $\mathbf{J}$ or $\mathbf{K}$, depending on condition). 
Table 3

Mean Fixation Times, in Seconds, During the Habituation Trials in

Experiment 3A and Preference Scores, in Percentages, for the Luminance Similarity Change Pattern During the Test Trials in Experiment 3A and Spontaneous Preference Trials in Experiment 3B

\begin{tabular}{|c|c|c|c|c|c|c|c|}
\hline & \multicolumn{4}{|c|}{ Habituation Trials } & \multirow{2}{*}{\multicolumn{3}{|c|}{$\begin{array}{c}\text { Test Trials: } \\
\text { Preference for } \\
\text { Luminance Organization }\end{array}$}} \\
\hline & \multicolumn{2}{|c|}{$\begin{array}{c}\text { First } \\
\text { Three Trials }\end{array}$} & \multicolumn{2}{|c|}{$\begin{array}{c}\text { Last } \\
\text { Three Trials } \\
\end{array}$} & & & \\
\hline & $M$ & $S D$ & $M$ & $S D$ & $M$ & $S D$ & $t$ \\
\hline \multicolumn{8}{|l|}{ Experiment $3 \mathrm{~A}$} \\
\hline $90^{\circ}$ rotation & 18.93 & 15.70 & 8.42 & 5.67 & 58.44 & 14.53 & $2.01^{*}$ \\
\hline Random & 20.71 & 14.29 & 7.87 & 6.38 & 56.23 & 8.32 & $2.59^{* *}$ \\
\hline Combined & 19.82 & 14.71 & 8.15 & 5.91 & 57.34 & 11.63 & $3.09^{* * *}$ \\
\hline \multicolumn{8}{|l|}{ Experiment 3B } \\
\hline $90^{\circ}$ rotation & & & & & 47.63 & 10.95 & -0.75 \\
\hline Random & & & & & 49.93 & 9.60 & -0.02 \\
\hline Combined & & & & & 48.78 & 10.14 & -0.59 \\
\hline
\end{tabular}

Preference scores during the test trials for the luminance similarity changed patterns are displayed in Table 3. An independent-samples $t$ test revealed no difference between the two luminance disruption conditions $\left[90^{\circ}\right.$ rotation and random conditions; $t(22)=-0.46, p>.20$, two-tailed]. The conditions were combined for our final analysis. Infants preferred to look at the pattern displaying a change in luminance to a significantly greater degree than the pattern displaying a change in UC $[t(23)=3.09, p<.01$, two-tailed].

The results of this experiment suggest that luminance similarity organization was more salient than UC was in this particular arrangement of stimuli for 6- to 7-montholds. However, before drawing a firm conclusion, it is important to rule out the possibility that the apparent greater salience of luminance over UC cues is not an artifact of a priori preferences between the test patterns.

\section{EXPERIMENT 3B}

As in Experiment 1B, we examined whether infants exhibit a spontaneous preference between the test patterns used in Experiment 3A. That is, infants in the present experiment were directly tested without any habituation for their preference between the test patterns used in Experiment $3 \mathrm{~A}$.

\section{Method}

Participants. Twenty-four 6- to 7-month-olds (9 males, 15 females; mean age $=203.25$ days, $S D=8.90$ ) participated.

Procedure. The procedure was exactly the same as that used in Experiment 3A, except that infants did not experience habituation trials and were directly exposed to the two 10 -sec test trials.

\section{Results and Discussion}

Infants' preference scores for the luminance change pattern are displayed in Table 3. An independent-samples $t$ test revealed no difference between the $90^{\circ}$ rotation and random conditions $[t(22)=0.48, p>.20$, two-tailed $]$. Consequently, the two conditions were combined in our main analyses. As in Experiment 1B, infants in the present experiment failed to exhibit a preference. Their mean preference score was not significantly different from the chance level of $50 \%$ $[t(23)=0.67, p>.20$, two-tailed], but it was significantly different from the novelty preference score exhibited by the infants in Experiment $3 \mathrm{~A}[t(46)=2.72, p<.01$, twotailed]. These results indicate that infants' performance in Experiment $3 \mathrm{~A}$ was due not to a priori preferences between the test stimuli but to the greater salience of changes in luminance-based than in UC-based organization.

\section{EXPERIMENT 4}

Both luminance and UC have been deemed highly important methods of organization by different researchers (e.g., Palmer \& Rock, 1994; Quinn et al., 1993). In Experiment $3 \mathrm{~A}$, luminance was shown to be more salient than UC. The present experiment was designed to further examine the salience of luminance over UC. Specifically, we examined whether UC organization was even encoded in the presence of luminance cues. Infants were habituated to the same stimuli used in Experiment 3A (which could be organized around both UC and luminance similarity organization) and were tested with novel patterns in which only the UC organization was altered.

\section{Method}

Participants. Twenty-four 6- to 7-month-olds (13 males, 11 females; mean age $=199.96$ days, $S D=10.10$ ) participated. One additional 6- to 7-month-old was excluded due to fussiness.

Stimuli. The habituation stimuli were identical to those employed in Experiment 3A. Infants were tested with the stimulus that they had viewed during habituation paired with the UC-altered stimuli from Experiment $3 \mathrm{~A}$; that is, all infants viewed the UC- and luminanceorganized habituation pattern paired with the pattern in which the bars were disconnected from the rows and columns of blocks and moved above or alongside the blocks (see Figure 4 for examples).

Procedure. The procedure was identical to that used in previous experiments.

\section{Results and Discussion}

A paired-samples $t$ test revealed a significant difference between the first three and last three habituation trials $[t(23)=7.34, p<.0001$, two-tailed]. Infants were therefore habituated to the stimuli.

Preference scores are displayed in Table 4. A $t$ test revealed that infants looked significantly longer at the novel 
$\mathbf{L}$

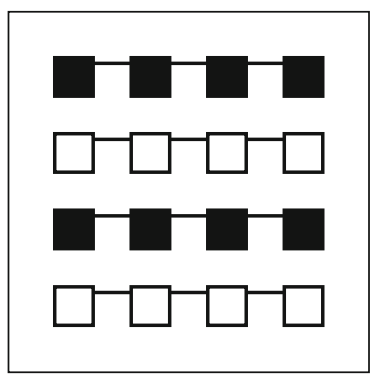

Habituation Stimulus
$\mathbf{L}$

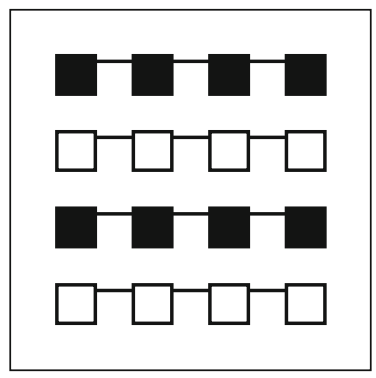

Habituation Stimulus
M

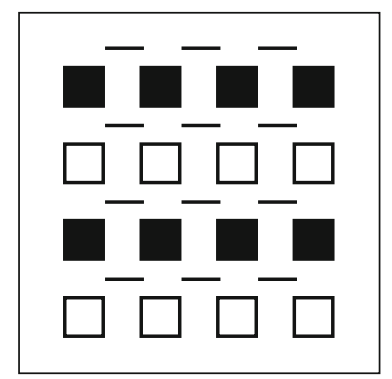

Change in UC

Figure 4. Examples of the stimuli used in Experiment 4. Infants were habituated to patterns of the kind shown in panel $L$ and then tested with patterns of the kind shown in panel $M$.

Table 4

Mean Fixation Times, in Seconds, During the Habituation Trials and Mean Preference Scores, in Percentages, for the Novel (Disconnected) Pattern in Experiment 4

\begin{tabular}{|c|c|c|c|c|c|c|c|}
\hline & \multicolumn{4}{|c|}{ Habituation Trials } & \multirow{2}{*}{\multicolumn{3}{|c|}{$\begin{array}{l}\text { Test Trials: } \\
\text { Preference for } \\
\text { Disconnected } \\
\text { Organization }\end{array}$}} \\
\hline & \multicolumn{2}{|c|}{$\begin{array}{c}\text { First } \\
\text { Three Trials }\end{array}$} & \multicolumn{2}{|c|}{$\begin{array}{c}\text { Last } \\
\text { Three Trials }\end{array}$} & & & \\
\hline & $M$ & $S D$ & $M$ & $S D$ & $M$ & $S D$ & $t$ \\
\hline Disconnected & 27.08 & 15.45 & 9.32 & 5.00 & 56.12 & 14.37 & $2.09^{* *}$ \\
\hline
\end{tabular}

${ }^{* *} p<.05$, two-tailed.

stimulus in which UC was altered than at the habituation pattern $[t(23)=2.09, p<.05$, two-tailed $] .{ }^{3}$ Infants were able to detect UC in the presence of luminance organization. This suggests that, whereas luminance organization cues may dominate UC cues, their presence is not so overwhelming as to mask those cues.

\section{GENERAL DISCUSSION}

In six experiments, we explored the salience of UC cues in relation to two other important organization cues. UC was more salient than shape similarity, to the extent that shape organization was not even detected by infants in the presence of UC in Experiments 1A and 2. Luminance similarity cues, however, affected infants' organization more than UC cues did, even though UC organization was detectable in the presence of luminance cues in Experiments $3 \mathrm{~A}$ and 4 . These results indicate that not all cues function in an equivalent manner in infancy. UC, although more salient than shape-based organization, is not necessarily the most influential organizational cue, at least among young infants (cf. Palmer \& Rock, 1994).

\section{The Relationship Between \\ Shape and UC Organization}

Infants in Experiment 1A found a change in UC to be more salient than a change in shape similarity. The outcome of this experiment is consistent with Palmer and Rock's (1994) assertion that UC is a fundamental mechanism of perceptual organization. Even though Experiment $1 \mathrm{~A}$ used a habituation approach rather than the RT approach normally employed in adult studies exploring the relationships between organizational principles, the results with infants are remarkably similar to those with adults (Han et al., 1999).

The finding in Experiment 2 that shape similarity cues are no longer detectable for infants when UC cues are present was unexpected because most models of infant perceptual development, from the most stringent (e.g., Kellman, 1993, 2000; Kellman \& Shipley, 1991) to those that are more flexible (e.g., Johnson \& Aslin, 1996; Needham, Baillargeon, \& Kaufman, 1997), predict that infants can organize on the basis of shape cues, in the age group that we worked with (6- to 7-month-olds). Also, research by Quinn and colleagues (Quinn \& Bhatt, 2006; Quinn et al., 2002) empirically demonstrated that 6- to 7-month-olds are sensitive to shape similarity organization. However, in the presence of UC, infants were unable to organize on the basis of shape similarity. This result attests to the strength of UC as an organizational cue in infancy. The findings also highlight the fact that shape similarity may require a greater amount of processing time and/or a greater depth of processing than UC (also see Quinn \& Bhatt, 2006).

The greater salience of UC cues relative to shape similarity cues may reflect the dichotomy of processing proposed in the models of Behrmann and Kimchi (2003) and Pomerantz (1981, 1983). Behrmann and Kimchi (see also Trick \& Enns, 1997) proposed that there are two stages in perceptual grouping: In the first stage, elements are clustered together on the basis of low-level perceptual grouping properties (e.g., proximity, closure, and luminance/ color) and reach a point of "belongingness" without the 
shape of the elements being identified. In the second stage, the shape of the elements is identified and the configural nature of the image composed of these individual elements is formed (Behrmann \& Kimchi, 2003; Kellman, 2003; Trick \& Enns, 1997). The Pomerantz (1981, 1983) model suggests that organizational tasks can be divided into those using Type $\mathrm{P}$ processing and those using Type $\mathrm{N}$ processing. Type $\mathrm{P}$ processing utilizes only the position of the elements in organizing an image, whereas Type $\mathrm{N}$ processing involves organizing on the basis of both the position and the characteristics of the individual elements making up the image, thus requiring more information for organization. This model suggests that Type $\mathrm{P}$ processing will be more readily achieved than Type N processing and, consequently, that organization based on Type P processing may be more salient than that done on the basis of Type N processing.

Since infants in Experiment 1A were habituated to an image that could be organized by both UC and shape, UC processing most likely took place more readily than did shape processing. This is because UC organization, by its very nature, does not require processing shape information, merely connected regions. Therefore, UC is most likely processed during the first stage of perceptual organization, as in Behrmann and Kimchi's (2003) model, and is a Type P task, according to the model proposed by Pomerantz $(1981,1983)$. Shape similarity is detected during the second stage, according to Behrmann and Kimchi's model, and is considered a Type N task. Presumably, therefore, processing based on shape similarity organization took place later in the stream of processing than processing based on UC, as suggested by the Behrmann and Kimchi model, and requires a greater amount and depth of processing, as suggested by the Pomerantz $(1981,1983)$ model. As a result, UC was more salient than shape similarity was in Experiment 1A. It should be noted, however, that the present experiments did not examine the order of processing of different kinds of perceptual organizational cues; hence, it is not clear that the relative salience of cues obtained in these experiments was related to the order of processing.

\section{The Relationship Between \\ UC and Luminance Similarity Cues}

The results of Experiment $3 \mathrm{~A}$ were not consistent with Pomerantz's $(1981,1983)$ Type N versus Type P model. In the test stimulus in which the UC cues were altered, the position of the lines was changed, thus modifying the global organization of the image. In the luminance similarity stimulus, the position of the bars remained intact, but the nature of the information represented by the squares was altered. According to the Type $\mathrm{N}$ versus Type $\mathrm{P}$ model, UC cues should have been more salient than luminance organization, but that is not what we found. Behrmann and Kimchi's (2003) model suggests that luminance and $\mathrm{UC}$ are types of organization that do not require the encoding of specific shape information; thus, both types of information should be encoded during the first stage of visual perception. Therefore, this model also does not ex- plain why luminance is more salient than UC. However, the Behrmann and Kimchi model, unlike the Pomerantz model, at least does not predict that UC would be more salient than luminance-based organization.

Kellman's (1993, 2000; Kellman \& Shipley, 1991) model of edge-insensitive (EI) versus edge-sensitive (ES) processing may also provide some explanation for the lack of salience of UC over luminance similarity, although this theory, like the Behrmann and Kimchi (2003) model, does not account for the superiority of luminance information. According to Kellman (1993, 2000), objects are identified in the EI stage on the basis of motion and other cues that do not rely on specific edge information to organize a pattern. Objects are identified in the ES stage by edge information, including orientation, closure, and texture, to name a few characteristics. This model further maintains that infants younger than 6 months of age have not developed the ES scheme of perceptual organization (Kellman, 1993, 2000; Kellman \& Shipley, 1991). Both luminance similarity and UC are EI processes, whereas shape similarity is an ES process. "Blobs" of lightness and darkness (luminance similarity) can be detected without perceiving the actual shape of the element, as can connectedness. Therefore, both luminance and UC organizations can result from the primitive EI system that enables quick organization into gestalt wholes. As evidenced by our results, however, the primitive luminance organization cues are more salient than the primitive UC cues.

\section{Conclusion}

The present research indicates a luminance-UC-shape salience hierarchy in infants' perceptual organization. We realize, however, that our findings do not necessarily indicate an absolute hierarchy, because the functioning of these organizational cues may differ depending on the salience of the specific values used in these different dimensions. Nevertheless, we believe that our findings reflect the general salience of these cues for the following reasons. In several studies now, we have found that shapesimilarity-based organization is more difficult for infants than other kinds of organization (e.g., Quinn \& Bhatt, 2005b; Quinn et al., 2002). This is the case, even though the particular elements used in these studies (e.g., Xs and $\mathrm{Os}$, Hs and Is, and diamonds and squares) are clearly discriminable to infants and the column/row organizations based on these elements are discerned by 6- to 7-monthold infants if competing cues are not present (Quinn \& Bhatt, 2005b; Quinn et al., 2002). Thus, our finding in Experiments $1 \mathrm{~A}-2$ that UC is more salient than shapebased organization is consistent with prior findings that shape-based grouping is one of the most difficult types of organization for infants.

In the case of the contrast between $\mathrm{UC}$ and luminance, we believe that the digital nature of connectedness (i.e., elements are either connected or not) makes it appropriate to contrast UC with luminance-based similarity differences to which infants have been previously shown to be sensitive. We believe that our choice of stimuli was validated by Experiment 4, which indicated that, 
even under conditions in which luminance is found to be more salient than UC, infants are able to discern the UC-based organization. In other words, despite the fact that infants could clearly see that elements were connected in our habituation patterns and the fact that UCbased cues led to columns/rows organizations, infants found the alternative organizations based on luminance similarity to be more compelling. Furthermore, the UC stimuli used in Experiments 3A-4 could not have been too weak, because similar cues dominated shape-based organization in Experiment 1A. Additional indication of the high salience of luminance cues (consistent with the findings of Experiment $3 \mathrm{~A}$ ) comes from the fact that there is evidence that even newborns are sensitive to luminance-based organization (Farroni et al., 2000). Thus, we believe that the differential significance of the three organizational cues exhibited in the study does reflect their general salience. However, it is possible that the relative salience of the cues studied in the present experiments may be altered depending on the particular values of the dimensions used, the contexts in which the relative salience of these cues are tested, and even the age of the participant. Thus, we do not view our findings as indicating an immutable hierarchy among the organizational cues.

Another issue concerns the nature of UC examined in the present experiments. Because the connected stimulus patterns were such that there was no change in the luminance of the connecting lines and the outer edges of the elements they connected, they met Palmer and Rock's (1994) definition of uniform connectedness. However, half of the elements in the present experiments had unfilled interiors (i.e., were outline elements), and this may have so affected the nature of connectedness that the organizational cue in the stimulus patterns may have seemed akin to element connectedness. This may have influenced the results of the study, because element connectedness is thought to be less of an entry-level organizational process than UC (Palmer \& Rock, 1994). Thus, despite the fact that, by definition, the connectedness cue used in the present experiments was $\mathrm{UC}$, which is thought to be an either/or kind of a cue (i.e., elements are either uniformly connected or not), there may be nuances to the degree and nature of connectedness that need to be further explored.

Finally, we note that the luminance-UC-shape salience hierarchy documented in this study mirrors the nature of development of sensitivity to these cues. As discussed above, there is some evidence suggesting that the use of luminance similarity to organize information may be available even at birth (Farroni et al., 2000). Sensitivity to $\mathrm{UC}$ is exhibited by at least 3 to 4 months of age (Hayden et al., 2006). However, it is sometime after 3 to 4 months of age that infants readily employ shape cues to organize perceptual information (Quinn \& Bhatt, 2005b; Quinn et al., 2002). Thus, the present findings, combined with prior studies that have documented the development of sensitivity to organizational cues, indicate a correspondence between the ontogenesis of sensitivity to different organizational cues and the relative salience of these cues at a particular age.

\section{AUTHOR NOTE}

This research was supported by grants from the National Science Foundation (BCS 0224240) and the National Institutes of Health (HD42451, HD-46526). We thank Michael Kavsek and Stephen Palmer for comments on an earlier version of the article. We also thank the infants and the parents who participated in this study. Correspondence concerning this article should be addressed to R. S. Bhatt, Psychology Department, University of Kentucky, Lexington, KY 40506-0044 (e-mail: rbhatt@email.uky.edu).

\section{REFERENCES}

Behrmann, M., \& Kimchi, R. (2003). What does visual agnosia tell us about perceptual organization and its relationship to object perception? Journal of Experimental Psychology: Human Perception \& Performance, 29, 19-42.

Bhatt, R. S., \& Bertin, E. (2001). Pictorial cues and three-dimensional information processing in early infancy. Journal of Experimental Child Psychology, 80, 315-332.

Duncan, J. (1984). Selective attention and the organization of visual information. Journal of Experimental Psychology: General, 113, 501-517.

Duncan, J., \& Humphreys, G. W. (1989). Visual search and stimulus similarity. Psychological Review, 96, 433-458.

Farroni, T., Valenza, E., Simion, F., \& Umiltà, C. (2000). Configural processing at birth: Evidence of perceptual organization. Perception, 29, 355-372.

Han, S., Humphreys, G. W., \& Chen, L. (1999). Uniform connectedness and classical Gestalt principles of perceptual grouping. Perception \& Psychophysics, 61, 661-674.

HaYden, A., BhatT, R. S., \& QuinN, P. C. (2006). Infants' sensitivity to uniform connectedness as a cue for perceptual organization. Psychonomic Bulletin \& Review, 13, 257-261.

Johnson, S. P., \& Aslin, R. N. (1996). Perception of object unity in infants: The roles of motion, depth, and orientation. Cognitive Development, 11, 161-180.

Kahneman, D., \& HeniK, A. (1981). Perceptual organization and attention. In M. Kubovy \& J. Pomerantz (Eds.), Perceptual organization (pp. 181-211). Hillsdale, NJ: Erlbaum.

Kahneman, D., \& Treisman, A. (1984). Changing views of attention and automaticity. In R. Parasuraman, D. R. Davies, \& J. Beatty (Eds.), Varieties of attention (pp. 29-62). New York: Academic Press.

Kellman, P. J. (1993). Kinematic foundations of infant visual perception. In C. Granrud (Ed.), Visual perception and cognition in infancy. Carnegie Mellon symposium on cognition (pp. 121-173). Hillsdale, NJ: Erlbaum.

Kellman, P. J. (2000). An update on Gestalt psychology. In B. Landau, J. Sabini, J. Jonides, \& E. Newport (Eds.), Perception, cognition, and language: Essays in honor of Henry and Lila Gleitman (pp. 158-190). Cambridge, MA: MIT Press.

Kellman, P. J. (2003). Visual perception objects and boundaries: A four-dimensional approach. In R. Kimchi, M. Behrmann, \& C. Olson (Eds.), Perceptual organization: Behavioral and neural perspectives (pp. 155-204). Mahwah, NJ: Erlbaum.

Kellman, P. J., \& Shipley, T. F. (1991). A theory of visual interpolation in object perception. Cognitive Psychology, 23, 141-221.

KIMCHI, R. (1998). Uniform connectedness and grouping in the perceptual organization of hierarchical patterns. Journal of Experimental Psychology: Human Perception \& Performance, 24, 1105-1118.

KIMCHI, R. (2000). The perceptual organization of visual objects: A microgenetic analysis. Vision Research, 40, 1333-1347.

Kramer, A. F., \& Watson, S. E. (1996). Object-based visual selection and the principle of uniform connectedness. In A. Kramer, M. Coles, \& G. Logan (Eds.), Converging operations in the study of selective attention (pp. 395-414). Washington, DC: American Psychological Association.

Moore, C. M., \& Egeth, H. (1997). Perception without attention: Evidence of grouping under conditions of inattention. Journal of Experimental Psychology: Human Perception \& Performance, 23, 339-352.

Needham, A., Baillargeon, R., \& Kaufman, L. (1997). Object segregation of infancy. In C. Rovee-Collier \& L. P. Lipsitt (Eds.), Advances in infancy research (Vol. 11, pp. 1-44). Norwood, NJ: Ablex. 
NeISSER, U. (1967). Cognitive psychology. East Norwalk, CT: AppletonCentury-Crofts.

Palmer, S., \& Rock, I. (1994). Rethinking perceptual organization: The role of uniform connectedness. Psychonomic Bulletin \& Review, 1, 29-55.

Pomerantz, J. R. (1981). Perceptual organization in information processing. In M. Kubovy \& J. R. Pomerantz (Eds.), Perceptual organization (pp. 141-180). Hillsdale, NJ: Erlbaum.

Pomerantz, J. R. (1983). Global and local precedence: Selective attention in form and motion perception. Journal of Experimental Psychology: General, 112, 512-540.

QuinN, P. C., \& BHATt, R. S. (2005a). Good continuation affects discrimination of visual pattern information in young infants. Perception \& Psychophysics, 67, 1171-1176.

QuinN, P. C., \& BHATT, R. S. (2005b). Learning perceptual organization in infancy. Psychological Science, 16, 511-515.

Quinn, P. C., \& Bhatt, R. S. (2006). Are some Gestalt principles deployed more readily than others during early development? The case of lightness versus form similarity. Journal of Experimental Psychology: Human Perception \& Performance, 32, 1221-1230.

Quinn, P. C., Bhatt, R. S., Brush, D., Grimes, A., \& Sharpnack, H. (2002). Development of form similarity as a Gestalt grouping principle in infancy. Psychological Science, 13, 320-328.

QuinN, P. C., Burke, S., \& Rush, A. (1993). Part-whole perception in early infancy: Evidence for perceptual grouping produced by lightness similarity. Infant Behavior \& Development, 16, 19-42.

Treisman, A. (1986). Properties, parts, and objects. In K. R. Boff, L. Kaufman, \& J. P. Thomas (Eds.), Handbook of perception and human performance (Vol. 2, pp. 1-46). New York: Wiley.

TRICK, L. M., \& ENNS, J. T. (1997). Clusters precede shapes in perceptual organization. Psychological Science, 8, 124-129.

Watson, S. E., \& Kramer, A. F. (1999). Object-based visual selection and perceptual organization. Perception \& Psychophysics, 61, 31-49.

Wertheimer, M. (1955). Gestalt theory. In W. D. Ellis (Ed.), A source book of Gestalt psychology (pp. 1-16). London: Routledge \& Kegan Paul. (Originally published in German)

\section{NOTES}

1. In the context of grouping, salience refers to the relative contribution of a cue for perceptual organization. In the present experiments, we operationally define the salience of a cue as the extent to which infants organize on the basis of this cue rather than on the basis of alternative, competing cues.

2. We chose not to examine infants' spontaneous preference between the test stimuli used in Experiment 2 because we believed that such a test would not be informative about the ability of infants to process organization on the basis of shape, in the presence of more salient UC cues. The lack of a preference during the test in Experiment 2 indicates that infants did not have a preference for the shape-changed stimuli (e.g., patterns $\mathrm{F}$ and $\mathrm{G}$ in Figure 2) because, otherwise, infants would have exhibited a preference for these stimuli on the basis of both a spontaneous preference and the shape change (if they had processed the shape change during habituation). A spontaneous preference in the opposite direction (e.g., for pattern E over F and/or G in Figure 2), even if it had existed, would not have been informative, because such a preference may not have been present after habituation in the case of the infants in Experiment 1A and thus would not have allowed us to conclude that infants can use shape information to organize when more salient UC cues are present. On the basis of this logic, and of the fact that UC was more salient than shape in Experiment 1A, our findings indicate that infants failed to organize on the basis of shape in the presence of UC cues.

3 . We chose not to conduct a test to examine infants' spontaneous preference among the test stimuli used in Experiment 4 because we believed that infants' preference for the UC-changed patterns (e.g., pattern M in Figure 4) during the test in this experiment indicates sensitivity to the UC cues in the presence of luminance cues, whether this preference was due to habituation or to spontaneous preference. This is because even a spontaneous preference for the UC-changed pattern must be based on changes in UC cues (compare patterns L and M in Figure 4). Thus, the findings from Experiments $3 \mathrm{~A}$ and $3 \mathrm{~B}$ that infants are more likely to base their organization on luminance cues than on UC cues when both cues are present, combined with the finding that infants discriminate between patterns that differ on the basis of UC cues (e.g., patterns L vs. M in Figure 4), indicate that, even though infants are sensitive to UC cues in the presence of luminance cues, the latter are more salient.

(Manuscript received April 6, 2008; revision accepted for publication July 3, 2008.) 